$\xi=-1$

\title{
Embedded design consideration for the implementation of food intake monitoring system: validated using LabVIEW software
}

\author{
C. Mahesh ${ }^{1}$ *, P. Kingston Stanley ${ }^{1}$, P. Vijaydaniel ${ }^{1}$, A. Saneevi Gandhi ${ }^{2}$ \\ ${ }^{1}$ Electronics and Instrumentation Engineering, Karunya Institute of Technology and Sciences \\ ${ }^{2}$ Electrical and Electronics Engineering, Karpagam College of Engineering \\ *Corresponding author E-mail:
}

\begin{abstract}
Memory loss is a common problem found among old people, the person may forget mealtime altogether due to memory loss. This paper proposes an idea for a monitoring system which will monitor the old people food intake from a remote place and generate an alarm locally. A wearable system is designed usingpiezoelectric sensor that will sense the trachea movement during food ingestion and produces an output signal with variations in the frequencies over time. Based on the detected frequency, food type is classified. Food classification algorithm is depends on Time-Frequency analysis of the piezoelectric output and the wearable design transmits the signals to PC via Bluetooth module. The received signal is processed by LabVIEW and accuracy is tested.
\end{abstract}

Keywords: Piezoelectric Sensor; Wearable Device; Food Intake Monitoring; Time-Frequency Analysis; LabVIEW.

\section{Introduction}

Forgetfulness is a disease, called Alzheimer, Commonly found in older adults. It is a degenerative disease that attacks the brain, causing impaired memory. The person may altogether forget their mealtime due to memory loss. It makes them incapable of caring for themselves. The inadequate food intake will further worsen the physical and mental health of the old people. So, it is necessary to monitor the food intake of old and sick people. Nowadays, our market shaped our way of living, having no time for caring elderly people. So that monitoring of the food intake by the old and sick people is very difficult. In this paper, we presented a wearable system to monitor the food intake of old and sick people.

Automatically monitoring the food intake of old people, without disturbing their daily habit is challenged one. The modern technology for patient monitoring is inaccurate and impractical due to the following reasons: 1) chances of false and undetection is more, for example food intake is detected based on hand movements [3]; 2) they interfere the day to day activity; 3 ) they are bulky and non wearable; 4) they are impractical, patient and old people cannot stay near the system [4]. There is a need for awearable monitoring system for food intake and to alert the person when they forget to eat food.

Many monitoring systems are available nowadays such as heart rate monitoring system, pressure monitoring system, glucose level monitoring system, but when one takes their food regularly, there won't be a need to use these kinds of monitoring systems. When we eat properly, the chances of getting thedisease is reduced. In this paper, it is mainly focused on a wearable food intake monitoring system which can be worn around the neck without disturbing our daily activities.
The proposed system will enable us to monitor the old and sick people who are alone in thehome, from being in our workplace. The monitoring system will monitor the food intake and it will send analert message to the concerned person through email or SMS. LabVIEW is used to process the acquired signal and to send analert message.

This paper is presents the content as follows. Related works are described in section(II). Food monitoring device is described in section (III). Food intake detection algorithm is described in section (IV). Results are discussed in section $\mathrm{V}$ and concluded in section (VI).

\section{Similar work}

Many sensors have been used in various ways to monitor food intake by detecting their swallow. Hoover et al. [3] introduce a method for detecting food intake by counting their bites taken during a meal. Orientation sensor was placed on the wrist to count the biting of food. Through this method, we can find the amount of food intake, but we can't identify whether they took medicine or food or water. At the same time when thehand is not rotated at a determined pattern, it will not count the food bite. So, thepossibility of wrong and undetection is more.

Sclabassi et al.performed food intake estimation using thevideobased algorithm. A wearable camera is used to record an individual's daily activity. Though the proposed algorithm shows good accuracy, implementing this in day to day life is really impractical. Similarly,sazonov et al.introduced food intake monitoring through chewing. Strain gauge sensor is used to detect food intake by sensing lower jaw movements. Since the device interfaced with a strain gauge transducer placed near the ear, which is not practically applicable for daily use. 
Ottaviano et al. [5] introduced food intake monitoring system through fiber optic endoscopic examination. In which endoscope is connected to avideo camera, DVD recorder, TV set. The setup will be bulky and non-wearable. Similarly,Limdi et al.introduced deglutition detection using themicrocontroller based device. Electrodes are placed on the patient's neck and EMG is performed to observe deglutition. The whole hardware design is bulky and the device is minimized to thehospital environment.

Detection of food intake have been explored by following several other methods [1],[2],[5]introduced food intake detection by sensing arm gestures using accelerometers and gyroscope sensors. Food intake is monitored by identifying the arm gestures associated with thehandling of cups, spoon, and plates. However, the way of eating does not disclose the amount of food intake. At the same time possibility of false detection is high. Controllers' analysis for non-linear system has been reported [17-29].

A piezoelectric sensor is a vibration based sensor element which generated voltages when the physical strain has applied and is used in numerous applications. The using ofpiezoelectricsensing element forthe monitoring of food intake is attempted by very few with several exceptions. In this paper, both food intake detection and classification of food intake is explained. Spectrogram which is mainly used for speech recognition and audio analysis is used to represent frequency changes over time. The main aim of the work is food intake monitoring using spectrogram analysis of sensed data.

\section{The wearable monitoring device}

Our food intake monitoring comprises of two parts, piezoelectric sensor part and LabVIEW part. Noise reduction, smoothening, frequency domain transform, guidance and feedback generation is performed using LabVIEW. This section describes the piezoelectric sensor part. Section IV will further discuss the algorithm implementation on the LabVIEW. Figure 1 shows the block diagram of the Food intake monitoring system.

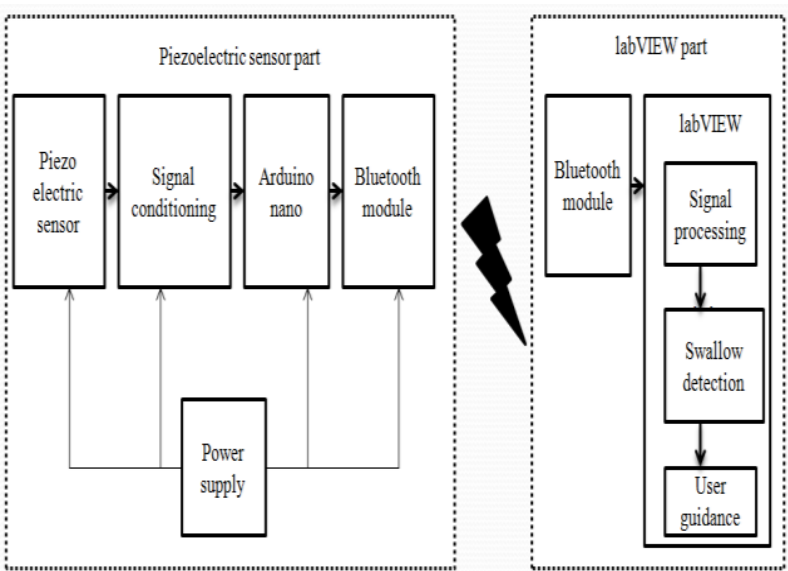

Fig. 1: Block Diagram of the Food Intake Monitoring System.

\subsection{Piezoelectric element}

A piezoelectric sensor is a vibration based sensor element which generated voltages when the physical strain has applied, this may be placed against the throat andthetrachea movement while swallow has represents the output of the sensor.The wearable device is designed by placing piezoelectric sensor inside small necklace shaped box with signal conditioning circuit. TL074 Transimpedance amplifier is used for signal conditioning. The conditioned signal is acquired by Arduino-Nano, which is transmitted to PC through HC-05 Bluetooth module. The PC receives the transmitted signal through Bluetooth dongle. LabVIEW is used to perform signal processing, smoothening and filtering. The user guidance and feedback also displayed using LabVIEW.

\subsection{Arduino Nano}

The Arduino-Nano is a small, breakout board integrated withATmega328 microcontrollerand it works with the usb power. The Nano has 6 ADC inputs, each of which provides 10 bits of resolution. The ATmega328 provides UART TTL (5v) serial communication, which is available on digital pins 0 (RX) and 1 (TX). The $\mathrm{RX}$ and TX LEDs will flash when data is being transmitted. In this paper, Arduino-Nano is used as DAQ to read analog signals from the piezoelectric sensor. The $5 \mathrm{v}$ from arduinoboard supplies power to the signal conditioning circuit and $\mathrm{HC}-05$ Bluetooth module.

\subsection{Bluetooth module}

$\mathrm{HC}-05$ is one of the Bluetooth module which usesserialprotocol in order to establish a transparent wireless connection and it can configure to work as slave or a master device. As a slave it cannot initiate aconnection, it can only accept theconnection. The HC-05 board has a $3.3 \mathrm{v}$ regulator that allows an input voltage of $3.3 \mathrm{v}$ to $6 \mathrm{v}$ but the TX and RX pins are still 3.3v. For HC-05 RX pin, it cannot be directly connected from arduino board. The voltage should be converted from $5 \mathrm{v}$ to $3.3 \mathrm{v}$ using avoltage divider and then it can be connected. Since the Arduino-Nano board will accept $5 \mathrm{v}$ as high, the HC-05 TX pin can be connected directly to the arduino RX pin.The default baud rate of HC-05 is 9600.The acquired signal from the wearable device is transmitted to the PC through Bluetooth module. In PC the signal is received through Bluetooth dongle. The signal will be transmitted and received without any error only when the baud rates are matched. To receive the transmitted signal through PC, right COM port must be specified. The COM port that is specified in VISA resource should match with the COM port displayed in device manager for Bluetooth connection.

\section{Implementation}

The food intake algorithm is implemented in LabVIEW. It is a platform that helps to design and develop a system. It accelerates the productivity with graphical programming syntax that makes it simple to create, visualize, and code the systems. It is difficult to monitor the food intake in acquired signal due to noise. Without signal processing, smoothening and filtering, we cannot differentiate the noise and the signal. The main objective of this paper is to detect each swallow during food intake. Guidance and user feedback is given based on the detected swallow. Figure 2 shows the food intake detection process.

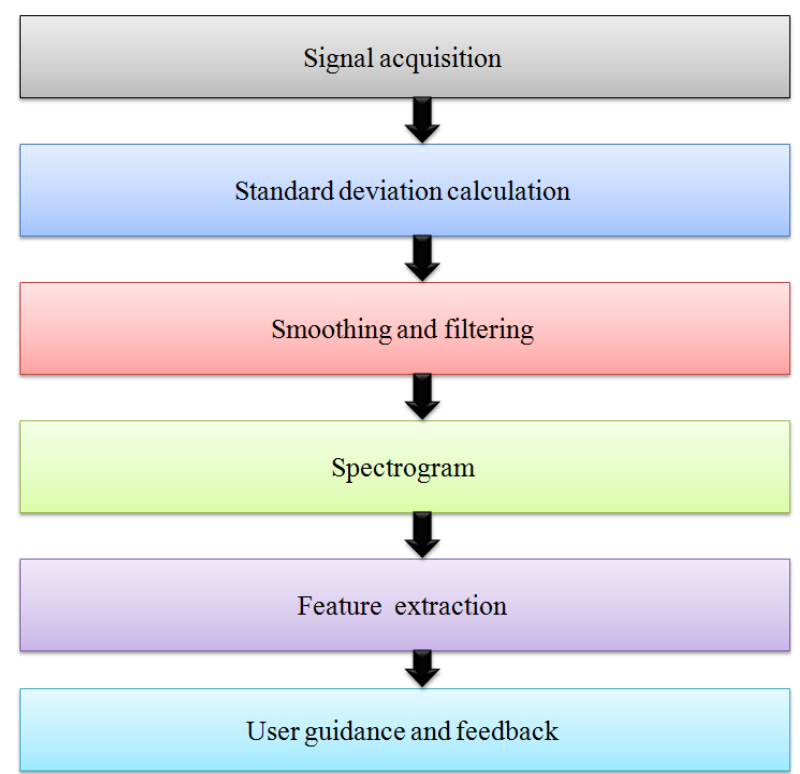

Fig. 2: Process Flow of Food Intake Monitoring System. 


\subsection{Standard deviation calculation}

Though the acquired signal is amplified using signal conditioning circuit, still it is difficult to view the clear signal due to the presence of noise. To reduce the noise content standard deviation is calculated for the acquired signal with asliding window. When we calculate standard deviation the noise is greatly suppressed. In standard deviation, the squared difference of each element from the mean value of the acquired signal is calculated and divided by $\mathrm{N}-1$. Within which range the signal is distributed can be obtained and theremaining unwanted signal is rejected. The standard deviation is calculated with the sliding window having window size, w. The value is calculated for the elements that lie within the window and then the window is shifted to next element.

$\mu=\frac{1}{N-1} \sum_{i=0}^{N-1} x_{i}$

$\sigma^{2}=\frac{1}{N-1} \sum_{i=0}^{N-1}\left(x_{i}-\mu\right)^{2}$

Where $\mu$ gives the mean value of the signal. Equation 2 gives the standard deviation value. This can be written in the following way

$\sigma^{2}=\frac{1}{N-1}\left[\sum_{i=0}^{N-1} x_{i}^{2}-\frac{1}{N}\left(\sum_{i=0}^{N-1} x_{i}^{2}\right)\right]$

$\sigma^{2}=\frac{1}{N-1}\left[\right.$ sum of squares $\left.-\frac{\text { sum }^{2}}{N}\right]$

The equation 2 is modified for the ease of calculating thestandard deviation with asliding window.

\subsection{Smoothing and filtering}

SavitzkyGolay filter is used for data smoothing, which is based on local least-squares polynomial approximation. During food intake, each swallow is represented as peaks in the waveform. The savitzkygolay filter reduces noise while maintaining the shape and height of waveform peaks. The polynomial is fitted to a set of input samples and then theapproximation is done. Initial central point is replaced by the computed value which is equivalent to discrete convolution with a fixed impulse response. The polynomial order should be less than the frame size.

\subsection{Spectrogram}

Spectrogram concept normally used for recognition of speech signal and it is the graphical representation of spectrum of frequencies in a signal. Generating spectrogram using the fast Fourier transform is a digital signal processing concepts. The time domain sample data are broken into small data or chunks, which overlaps and then transformed to calculate the amplitude of the frequency spectrum.Once food intake is detected; thespectrogram is calculated around each signal. Spectrogram of a signal can be calculated by computing the squared magnitude of the STFT of the signal in the following way.

$\operatorname{spectrogram}(t, w)=|\operatorname{STFT}(t, w)|^{2}$

In STFT, the signal to be transformed is multiplied by the window function. We used hanning window of window size 32. The equation is mathematically written as,

$\operatorname{STFT}\{x(t)\}(\tau, \omega) \equiv X(\tau, \omega)=\sum_{t=-\infty}^{\infty} x[t] \omega[t-\tau] e^{-j \omega t}$

\subsection{Feature extraction and user guidance}

It is possible to monitor the food intake using spectrogram. Once the spectrogram is obtained, to give user guidance and feedback feature extraction is necessary. Spectrograms are 2D matrices. Interpreting $2 \mathrm{~d}$ spectrograms cannot be done directly. In LabVIEW to extract useful 1D information,time-frequency analysis tools are used. The mean instantaneous frequency is computed to help with further feature extraction. The center of gravity of the power spectrum of the signal is described by the mean frequency computation. In food intake monitoring, the food type is classified based on the frequency.

The main objective of this food monitoring is to help old and sick people. Since they are incapable to take caring of themselves, to make them eat their tablets and food, this wearable design can be used. While taking tablets they will be swallowing it without chewing so taking tablet will come under high frequency. But while taking food they will chew well and swallow it so food intake will come with slow frequency. To differentiate food intake and tablet intake, thresholding is used. The frequency when it crosses the threshold value, it is noted as tablet intake, while less than thethreshold value is noted as food intake. Based on the intake, user guidance and feedback is displayed in LabVIEW. Based on this, when they didn't take the food and tablet, the feedback can be seen in LabVIEW and the alert message can be sent to the caretaker through SMS or E-mail.

\section{Result}

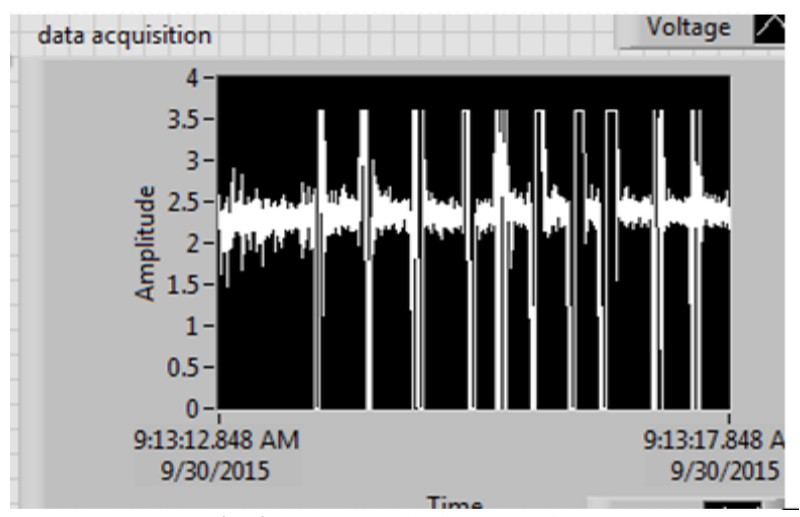

Fig. 3: Data Acquisition Waveform.

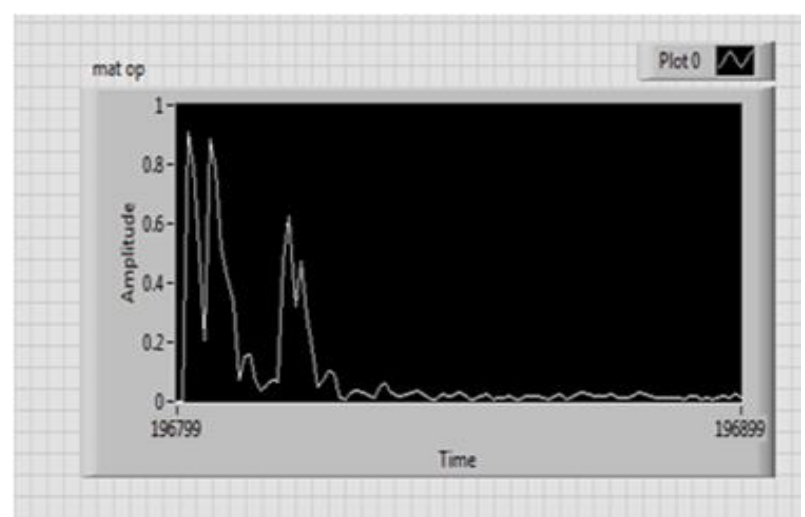

Fig. 4: Standard Deviated Through Sliding Window Waveform.
) 


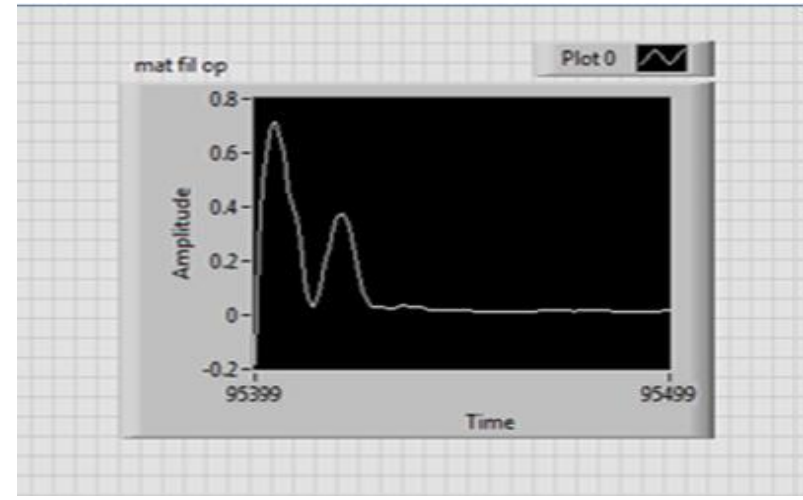

Fig. 5: Smoothed Waveform.

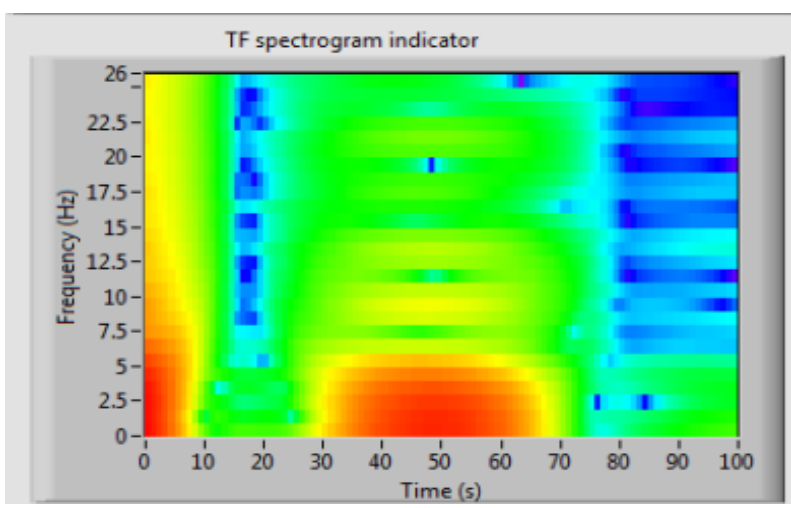

Fig. 6: Spectrogram Generated in LabVIEW.

(A)

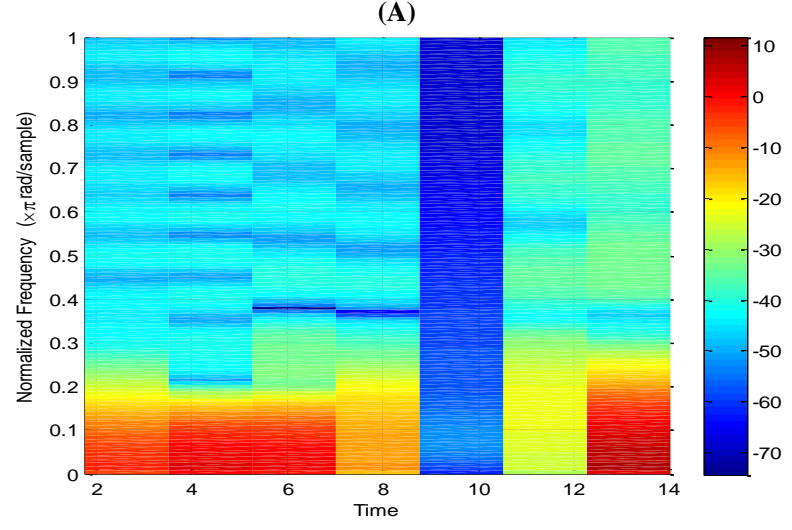

(B)

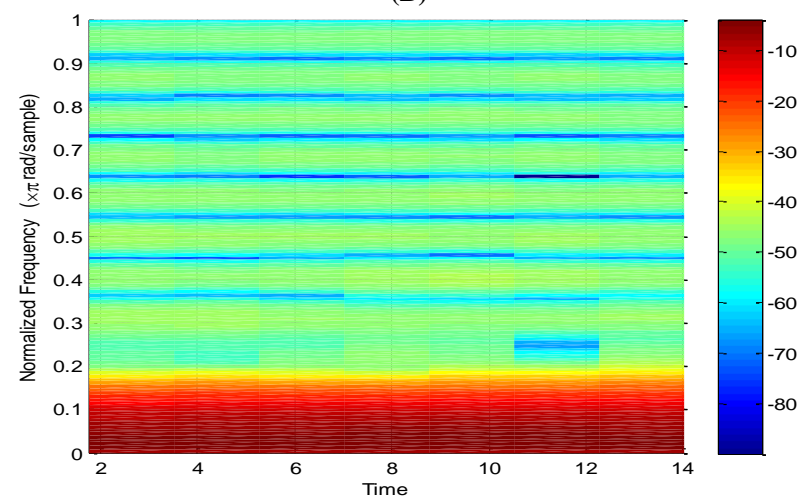

Fig. 7: Spectrogram Generated In MATLAB A) High Frequency, B) Low Frequency.

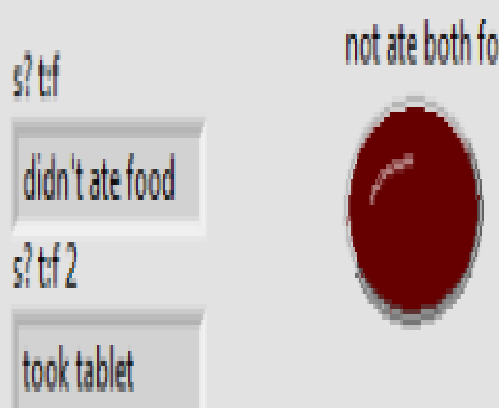

Fig. 8: User Guidance and Feedback when Tablet Alone is Taken.

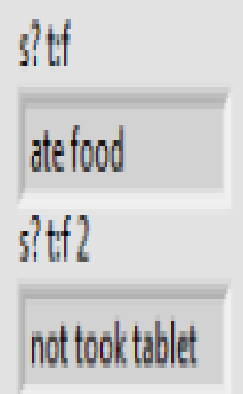

not ate both food and tablet

Fig. 9: User Guidance and Feedback when Food Alone is Taken.

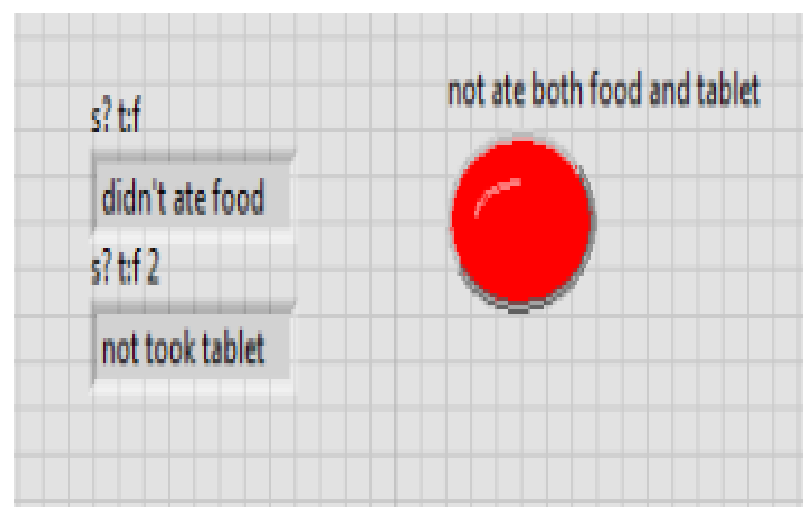

Fig. 10: User Guidance and Feedback when both Food and Tablet is Not Taken.

\section{Conclusion}

In this paper, we performed food intake detection by detecting each swallow of food using spectrogram generation of piezoelectric signals. We have developed the wearable device that successfully distinguishes the food and tablet intake. Our future work is to design the food intake monitoring system using the embedded cloud.

\section{References}

[1] M. Aboofazeli and Z. Moussavi, "Automated classification of swallow-ing and breadth sounds," in Proc. 26th Annu. Int. Conf. IEEE Eng. Med.Biol. Soc. (IEMBS), vol. 2, Sep. 2004, pp. 3816-3819.

[2] N. Alshurafa, H. Kalantarian, M. Pourhomayoun, S. Sarin, J. Liu, and Sarrafzadeh, "Non-invasive monitoring of eating behavior using spectrogram analysis in a wearable necklace," in Proc. IEEE EMBSHealthcare Innov. Point Care Technol. (HIPT), Oct. 2014, pp. 71-74.

[3] Y. Dong, A. Hoover, and E. Muth, "A device for detecting and counting bites of food taken by a person during eating," in Proc. IEEE Int. Conf. Bioinformat. Biomed. (BIBM), Nov. 2009, pp. 265-268. https://doi.org/10.1109/BIBM.2009.29. 
[4] O. Amft and G. Tröster, "Methods for detection and classification of normal swallowing from muscle activation and sound," in Proc. 1st Int.Conf. Pervasive Comput. Technol. Healthcare (ICST), Nov. 2006, pp. 1-10. https://doi.org/10.1109/PCTHEALTH.2006.361624.

[5]

O.Amft, H. Junker, and G. Troster, "Detection of eating and drinking arm gestures using inertial body-worn sensors," in Proc. 9th IEEE Int.Symp. Wearable Comput., Oct. 2005, pp. 160-163. https://doi.org/10.1109/ISWC.2005.17.

[6] H. Kalantarian, N. Alshurafa, T. Le, and M. Sarrafzadeh, "Monitoring eating habits using a piezoelectric sensor-based necklace," Comput. Biol.Med., vol. 58, pp. 46-55, Mar. 2015 https://doi.org/10.1016/j.compbiomed.2015.01.005.

[7] H. Kalantarian, N. Alshurafa, and M. Sarrafzadeh, "A wearable nutrition monitoring system," in Proc. IEEE 11th Int. Conf. Wearable Implant.Body Sensor Netw., Jun. 2014, pp. 75-80. https://doi.org/10.1109/BSN.2014.26.

[8] T. Klap and Z. Shinar, "Using piezoelectric sensor for continuouscontact-free monitoring of heart and respiration rates in real-life hospital settings," in Proc. Comput. Cardiol. Conf. (CinC), Sep. 2013, 671-674.

[9] A. K. Limdi, M. J. McCutcheon, E. Taub, W. E Whitehead, and E. W. Cook, III, "Design of a microcontroller-based device for deglutition detection and biofeedback," in Proc. Annu. Int. Conf. IEEE Eng.Med. Biol. Soc., Images 21st Century, vol. 5, Nov. 1989, pp. 1393-1394.

[10] O. Makeyevet al., "Recognition of swallowing sounds using timefrequency decomposition and limited receptive area neural classifier," in Applications and Innovations in Intelligent Systems XVI, T. Allen, R. Ellis, and M. Petridis, Eds. London, U.K.: SpringerVerlag, 2009, 33-46.

[11] M. Nagae and K. Suzuki, "A neck mounted interface for sensing the swallowing activity based on swallowing sound," in Proc. Annu. Int.Conf. Eng. Med. Biol. Soc. (EMBC), Aug./Sep. 2011, pp. 5224-5227. https://doi.org/10.1109/IEMBS.2011.6091292.

[12] A. Savitzky and M. J. E. Golay, "Smoothing and differentiation of data by simplified least squares procedures," Anal. Chem., vol. 36, no. 8, pp. 1627-1639, 1964. https://doi.org/10.1021/ac60214a047.

[13] E. S. Sazonov and J. M. Fontana, "A sensor system for automatic detection of food intake through non-invasive monitoring of chewing," IEEE Sensors J., vol. 12, no. 5, pp. 1340-1348, May 2012. https://doi.org/10.1109/JSEN.2011.2172411.

[14] D. Sussillo, A. Kundaje, and D. Anastassiou, "Spectrogram analysis of genomes," EURASIP J. Adv. Signal Process. vol. 2004, pp. 2942, Jan. 2004. https://doi.org/10.1155/S1110865704310048.

[15] N. Yao, R. J. Sclabassi, Q. Liu, and M. Sun, "A video-based algorithm for food intake estimation in the study of obesity," in Proc. IEEE 33rdAnnu. Northeast Bioeng. Conf. (NEBC), Mar. 2007, pp. 298-299.

[16] F.G.D’Ottaviano, T.A LinharesFilho, H.M.T. de Andrade,P.C.L.Alves, and M.S.G. Rocha,'Fiberoptic endoscopy evaluation of swallowing in patients with amyotrophic lateral sclerosis ," Brazilian j.Otorhinolaryngol,. vol. 79, no.3,pp. 349353,May/Jun.2013. https://doi.org/10.5935/1808-8694.20130061.

[17] R. Kalaivani, K. Ramash Kumar, S. Jeevananthan, "Implementation of VSBSMC plus PDIC for Fundamental Positive Output Super Lift-Luo Converter," Journal of Electrical Engineering, Vol. 16, Edition: 4, 2016, pp. 243-258.

[18] K. Ramash Kumar,'Implementation of Sliding Mode Controller plus Proportional Integral Controller for Negative Output Elementary Boost Converter," Alexandria Engineering Journal (Elsevier), 2016, Vol. 55, No. 2, pp. 1429-1445. https://doi.org/10.1016/j.aej.2016.03.027.

[19] P. Sivakumar, V. Rajasekaran, K. Ramash Kumar, "Investigation of Intelligent Controllers for Varibale Speeed PFC Buck-Boost Rectifier Fed BLDC Motor Drive," Journal of Electrical Engineering (Romania), Vol.17, No.4, 2017, pp. 459-471

[20] K. Ramash Kumar, D.Kalyankumar, DR.V.Kirbakaran” An Hybrid Multi level Inverter Based DSTATCOM Control, Majlesi Journal of Electrical Engineering, Vol. 5. No. 2, pp. 17-22, June 2011, ISSN: 0000-0388.

[21] K. Ramash Kumar, S. Jeevananthan, “A Sliding Mode Control for Positive Output Elementary Luo Converter," Journal of Electrical Engineering, Volume 10/4, December 2010, pp. 115-127.

[22] K. Ramash Kumar, Dr.S. Jeevananthan,” Design of a Hybrid Posicast Control for a DC-DC Boost Converter Operated in Continuous Conduction Mode" (IEEE-conference PROCEEDINGS OF ICETECT 2011), pp-240-248, 978-1-4244-7925-2/11.

[23] K. Ramash Kumar, Dr. S. Jeevananthan," Design of Sliding Mode Control for Negative Output Elementary Super Lift Luo Converter
Operated in Continuous Conduction Mode", (IEEE conference Proceeding of ICCCCT-2010), pp. 138-148, 978-1-4244-7768-5/10.

[24] K. Ramash Kumar, S. Jeevananthan, S. Ramamurthy" Improved Performance of the Positive Output Elementary Split Inductor-Type Boost Converter using Sliding Mode Controller plus Fuzzy Logic Controller, WSEAS TRANSACTIONS on SYSTEMS and CONTROL, Volume 9, 2014, pp. 215-228.

[25] N. Arunkumar, T.S. Sivakumaran, K. Ramash Kumar, S. Saranya, "Reduced Order Linear Quadratic Regulator plus Proportional Double Integral Based Controller for a Positive Output Elementary Super Lift Luo-Converter," JOURNAL OF THEORETICAL AND APPLIED INFORMATION TECHNOLOGY, July 2014. Vol. 65 No.3, pp. 890-901.

[26] Arunkumar, T.S. Sivakumaran, K. Ramash Kumar, "Improved Performance of Linear Quadratic Regulator plus Fuzzy Logic Controller for Positive Output Super Lift Luo-Converter," Journal of Electrical Engineering, Vol. 16, Edition:3, 2016, pp. 397-408.

[27] Kingston Stanley P, Sanjeevi Gandhi A "INDUSTRIAL EFFLUENT WATER PH NEUTRALIZATION CONTROLLER DESIGN USING LABVIEW AND MATLAB" International Journal of Pure and Applied Mathematics Volume 117 No. 10. (2017), 13-17 doi: 10.12732/ijpam.v117i10.4

[28] Vijay Daniel P, Sanjeevi Gandhi A "DESIGN OF MODELING AND CONTROL OF TEMPERATURE PROCESS INDOWNDRAFT BIOMASS GASIFIER: SIMULATION STUDIES" International Journal of Pure and Applied Mathematics Volume 117 No. 10. (2017), 19-23 doi: 10.12732/ijpam.v117i10.3

[29] Vijay Daniel P, Sanjeevi Gandhi A "Design of Mathematical Modelling and Control of Downdraft Biomass Gasifier" International Journal of Control and Automation Vol. 10, No. 11 pp 175 (2017) https://doi.org/10.14257/ijca.2017.10.11.16

[30] S.V.Manikanthan and D.Sugandhi "Interference Alignment Techniques For Mimo Multicell Based On Relay Interference Broadcast Channel" International Journal of Emerging Technology in Computer Science \& Electronics (IJETCSE) ISSN: 0976-1353 Volume7, Issue 1-MARCH 2014.

[31] T. Padmapriya and V. Saminadan, "Distributed Load Balancing for Multiuser Multi-class Traffic in MIMO LTE-Advanced Networks", Research Journal of Applied Sciences, Engineering and Technology (RJASET) - Maxwell Scientific Organization, ISSN: 2040-7459; eISSN: 2040-7467, vol.12, no.8, pp: 813-822, April 2016.

[32] S Nazeer Hussain, K Hari Kishore "Computational Optimization of Placement and Routing using Genetic Algorithm" Indian Journal of Science and Technology, ISSN No: 0974-6846, Vol No.9, Issue No.47, page: 1-4, December 2016.

[33] K. Ramash Kumar, Dr. S. Jeevananthan,” Design of Sliding Mode Control for Negative Output Elementary Super Lift Luo Converter Operated in Continuous Conduction Mode", (IEEE conference Proceeding of ICCCCT-2010), pp. 138-148, 978-1-4244-7768-5/10. 